\title{
Reply to comment of Prof. Artur Czekierdowski
}

\author{
Grzegorz Jakiel \\ Professor W. Orlowski Memorial Hospital, The Centre of Postgraduate Medical Education, Warsaw, Poland
}

\section{Dear Editor,}

I have read the letter by Professor Czekierdowski regarding the publication by Ciebiera et al. "Case report of ovarian torsion mimicking ovarian cancer as an uncommon late complication of laparoscopic supracervical hysterectomy" with great interest as it contains numerous significant statements that to some extent question our findings; therefore, I would like to refer to the comments as they were presented in the letter:

- A black and white ultrasound scan was published because the scanner printer had some technical limitations and not because we had decided not to assess tumour blood supply; the scan plays only a representative function because we described the lesion in detail and we did not assume that our readers would want verify whether it was correct.

- I am in strong opposition to the interpretation by Prof. Czekierdowski that age is a determining factor for the woman's menopausal age. The age 50+ years is only an estimate with regard to the incidence of menopause in the population and this variable is presented in the literature in this context. In this case the oestradiol levels were measured (they were below the laboratory detection threshold - below 11.8 $\mathrm{pg} / \mathrm{ml}$ and $\mathrm{FSH} 71.5 \mathrm{mlU} / \mathrm{ml}$ ), therefore undoubtedly the patient was menopausal, whereas the He4 protein levels of $83.1 \mathrm{pmol} / \mathrm{l}$ in a 46-year-old female patient were definitely elevated. Undoubtedly, there has been a discussion in the literature about whether the cut-off point for this test can be the same for the whole population or whether it should vary depending on the age group (irrespective of the hormone status), but this discussion is far from conclusive [ 1 , 2]. Nonetheless, I have not found any studies regarding a linear correlation between the Risk of Ovarian Malignancy Algorithm (ROMA) test results and the risk level in the literature. There is only mention of the cut-off point used to divide the population into low- and high-risk groups, so the expression "only slightly increased risk" used by Prof. Czekierdowski seems not to be accurate.

- The Authors know and understand the International Ovarian Tumour Analysis (IOTA) terms and definitions, and also two of them have gained an IOTA
Certificate. In order to ensure that ovarian cancer patients receive appropriate treatment, an accurate characterisation of any adnexal mass that requires surgery is pivotal to improving the outcome of this disease [3]. We assume that the problem with preoperative diagnosis of the presented case is related to the use of some subjective descriptors like the presence or absence of acoustic shadows on the image of the tumour. Using logistic regression model 2 (LR2) or ADNEX model we could get obtain a different risk of malignancy if there is a scored presence (or absence) of acoustic shadows. Subjective assessment by experienced examiners is generally accepted to be the best way to classify adnexal masses prior to surgery. [3] An advantage of the LR2 or Simple Rules models over subjective assessment is their objectivity and simplicity, which facilitates their use by ultrasonographers with different backgrounds and various levels of experience. [5, 6]. The vascularity of the mass and the tumour malignant feature M4 was assessed based on some other scans that were not presented in the article. Moreover, masses in which the Simple Rules yield an inconclusive result can be classified using subjective assessment by an experienced ultrasound operator or, given the high prevalence of malignancy in this group, they can all be classified as malignant to increase the sensitivity for ovarian cancer $[3,7]$. According to Timmerman [4] we could mention that the B-feature B1 (unilocular cyst) was most predictive of a benign tumour, while the B-feature B3 (acoustic shadows) was least predictive; additionally, the $M$-feature M2 (ascites) was most predictive of malignancy, while the M-feature M4 (irregular multilocular solid tumour with largest diameter > 100 $\mathrm{mm}$ ) was least predictive. Evidence from the latest (2016) meta-analysis shows that, although RMI is used most commonly, both subjective assessment and simple rules, added with subjective assessment in case simple rules are inconclusive, show a superior test accuracy. The choice between using subjective assessment or simple rules can be made depending on the expertise present in the hospital. [3].

- Another fragment of the letter refers to laparoscopic supracervical hysterectomy (LSH) complications as- 
sociated with morcellation compared to low significance of torsion of the remaining adnexa. Prof. Czekierdowski suggests development of serious complications associated with morcellation and the risk of dissemination of undiagnosed neoplasm or disseminated myomatosis. In my opinion, these comments are due to the hysteria observed in the USA when there was one case of an undiagnosed myoma in a morcellated uterus in a prominent patient. The latest reports, including the paper by Smits et al. quoted in our work, do not confirm the risk associated with this method. Torsion of the remaining adnexa is listed among complications of hysterectomy, and its significance may be greater with the increasing number of surgeries when ovaries remain. The authors intended to draw attention to the fact that this situation is possible and not to demonstrate that this is the leading long-term LSH complication. The decision to open the abdominal cavity with a lower midline incision that had been made previously is made relatively rarely at the site with the $86 \%$ rate of laparoscopic surgeries (including oncological ones); it was a result of a risk-benefit assessment, and in our opinion it might have not been replaced with microlaparotomy, as Prof. Czekierdowski suggests, because in such a case the rule states that such an opening should be made so that it would not be necessary to decrease a tumour in the peritoneal cavity. Endobag evacuation was not possible due to preoperative decisions when the solid character of the lesion was taken into account.

Yours sincerely,

Professor Grzegorz Jakiel MD, PhD on behalf of the Authors

\section{References}

1. Moore RG, Miller MC, Eklund EE, et al. Serum levels of the ovarian cancer biomarker HE4 are decreased in pregnancy and increase with age. Am J Obstet Gynecol 2012; 206: 349.e1-7.

2. Moore RG, McMeekin DS, Brown AK, et al. A novel multiple marker bioassay utilizing HE4 and CA125 for the prediction of ovarian cancer in patients with a pelvic mass. Gynecol Oncol 2009; 112: 40-46.

3. Meys EM, Kaijser J, Kruitwagen RF. Subjective assessment versus ultrasound models to diagnose ovarian cancer: A systematic review and meta-analysis. Eur J Cancer 2016; 58: 17-29.

4. Timmerman D, Van Calster B, Testa A, et al. Predicting the risk of malignancy in adnexal masses based on the Simple Rules from the International Ovarian Tumor Analysis group. Am J Obstet Gynecol 2016; 214: 424-437.

5. Nunes N, Ambler G, Hoo WL, et al. A prospective validation of the IOTA logistic regression models (LR1 and LR2) in comparison to subjective pattern recognition for the diagnosis of ovarian cancer. Int J Gynecol Cancer 2013; 23: 1583-1589.

6. Alcazar JL, Pascual MÁ, Olartecoechea B, et al. IOTA simple rules for discriminating between benign and malignant adnexal masses: a prospective external validation. Ultrasound Obstet Gynecol 2013; 42: 467-471.

7. Kaijser J, Bourne T, Valentin L, et al. Improving strategies for diagnosing ovarian cancer: a summary of the International Ovarian Tumor Analysis (IOTA) studies. Ultrasound Obstet Gynecol 2013; 41: 9-20. 